\title{
Correction to: Cell adhesion molecules, plasminogen activator inhibitor type 1, and metabolic syndrome in patients with psoriasis
}

\author{
Guilherme Gomes Teixeira ${ }^{1}$ Naiara Lourenço Mari ${ }^{1}$ Jaqueline Costa Castardo de Paula ${ }^{1}$. \\ Camila Cataldi de Alcantara ${ }^{1}$ - Tamires Flauzino ${ }^{1}$ Marcell Alysson Batisti Lozovoy ${ }^{1,2}$ - Ligia Márcia Mário Martin ${ }^{3,4}$. \\ Edna Maria Vissoci Reiche ${ }^{1,2} \cdot$ Michael Maes $^{5,6} \cdot$ Isaias Dichi $^{4} \cdot$ Andréa Name Colado Simão ${ }^{1,2}$ (1)
}

Published online: 8 January 2020

(c) Springer Nature Switzerland AG 2020

\section{Correction to: Clinical and Experimental Medicine https://doi.org/10.1007/s10238-019-00595-2}

The original version of this article unfortunately contained a mistake. Camila Cataldi de Alcantara was not listed among the authors. The corrected author group should be:

Guilherme Gomes Teixeira · Naiara Lourenço Mari - Jaqueline Costa Castardo de Paula - Camila Cataldi de Alcantara - Tamires Flauzino - Marcell Alysson Batisti Lozovoy · Ligia Márcia Mário Martin · Edna Maria Vissoci Reiche · Michael Maes · Isaias Dichi · Andréa Name Colado Simão

The original article has been corrected.

The original article can be found online at https://doi.org/10.1007/ s10238-019-00595-2.

Andréa Name Colado Simão

deianame@yahoo.com.br

Guilherme Gomes Teixeira

gtggomes@hotmail.com

Naiara Lourenço Mari

naiaralm@hotmail.com

Jaqueline Costa Castardo de Paula

costacastardo@gmail.com

Tamires Flauzino

t.flauzino@hotmail.com

Marcell Alysson Batisti Lozovoy

marcell_lozovoy@hotmail.com

Ligia Márcia Mário Martin

ligiadermato@gmail.com

Edna Maria Vissoci Reiche

reiche@sercomtel.com.br

Michael Maes

dr.michaelmaes@hotmail.com
Publisher's Note Springer Nature remains neutral with regard to jurisdictional claims in published maps and institutional affiliations.
Isaias Dichi

dichi@sercomtel.com.br

1 Research Laboratory in Applied Immunology, University of Londrina, Londrina, Brazil

2 Department of Pathology, Clinical Analysis and Toxicology, University of Londrina, Avenida Robert Koch, No. 60, Londrina, PR CEP: 86038-440, Brazil

3 Department of Dermatology, University of Londrina, Londrina, PR, Brazil

4 Department of Internal Medicine, University of Londrina, Londrina, PR, Brazil

5 IMPACT Strategic Research Centre, School of Medicine, Deakin University, Geelong, VIC, Australia

6 Department of Psychiatry, Faculty of Medicine, Chulalongkorn University, Bangkok, Thailand 